\title{
MOLECULAR DYNAMICS, SMOOTH PARTICLE APPLIED MECHANICS, AND CLAUSIUS' INEQUALITY
}

\author{
WM. G. HOOVER \\ University of California at Davis/Livermore and Lawrence Livermore National Laboratory \\ Livermore, California 94551-7808 USA 510-422-9787 \\ (hoover@wente. 1lnl.gov)
}

\begin{abstract}
Recent developments in molecular dynamics furnish new interconnections among three classical fields: particle mechanics, continuum mechanics, and thermodynamics. The resulting links clarify the importance of Lyapunov instability to irreversibility.

PACS Numbers: 03.20.+i 05.60.+w 46.10.+z
\end{abstract}

\section{INTRODUCTION}

The paradoxical coexistence of time-reversible mechanics with the second-law inequalities of thermodynamics has troubled physicists for more than 100 years. These two approaches to understanding, the one theoretical and the other experimental in outlook, were joined by a third route to knowledge, computer simulation, at the time of the Second World War. Recent extensions of the computational approach, born of a desire to simulate nonequilibrium processes, can be analyzed from Gibbs' phase-space point of view. This approach leads quite naturally to a microscopic mechanical analog of the macroscopic Clausius' Inequality [1,2]:

$$
\oint \dot{Q} / T<0 \text {. }
$$

This macroscopic inequality summarizes the thermodynamic observation that cyclic processes generate heat. The microscopic mechanical analog of this inequality,

$$
\langle d \ln \otimes / d t\rangle<0,
$$

reflects the same observation, but with the rate of heat generation replaced by the relative expansion rate of the phase-space hypervolume $\otimes$. In the microscopic case the need for time averaging is indicated explicitly by the angle brackets $<>$.

A recent particle approach to macroscopic simulation, smooth particle applied mechanics [3, 4], has revealed new links between the reversibility paradox which separates classical mechanics from thermodynamics, and promises assistance in understanding the irreversibility inherent in turbulent flows. Here we discuss the concepts of temperature, heat transfer, Lyapunov instability, and turbulence, in order to enhance the understanding of the connections among them.

\section{TEMPERATURE, THERMOSTATS, AND HEAT TRANSFER}

Temperature requires a thermometer, as is emphasized by Muschik's term, "contact temperature", though the zeroth law of thermodynamics makes it plain that the exact nature of the thermometer is unimportant at equilibrium. Away from equilibrium, using simplicity as a guide, it is natural to use an ideal gas of light particles, capable of un- 
correlated impulsive collisions, to define local temperature. Such an ideal-gas thermometer, through impulsive collisions, measures the kinetic energy of the degrees of freedom with which it interacts. The resulting definition of ideal-gas temperature follows from kinetic theory. For a single Cartesian degree of freedom

$$
k T \equiv\left\langle m \dot{x}^{2}\right\rangle .
$$

The arbitrariness of such a choice can be seen by considering the fact that two general nonequilibrium systems, with different nonequilibrium velocity distributions, but sharing a common value of the kinetic temperature $T$, would typically exchange energy between themselves, if they were able to interact impulsively, while neither nonequilibrium system would have a net energy exchange with an idealized ideal-gas thermometer set at their common kinetic temperature $T$.

The kinetic-theory definition of an ideal-gas thermometer is one of the classical approaches to thermodynamics, and is not only the simplest, but also the one most closely tied to computer simulation. The kinetic-theory definition of temperature can be embedded into the framework of classical mechanics through the use of Hamilton's Principle of Least Action [5], That Principle requires that particle trajectories minimize the action integral while simultaneously satisfying all externally-imposed constraints. A thermal constraint requires that the kinetic energy of any thermostatted set of degrees of freedom remains constant. Such a phase-space constraint reduces, by one, the number of degrees of freedom describing the system, and results in a new thermostatted equation of motion for the constrained coordinates:

$$
m \ddot{r}=F(r)-\zeta m \dot{r} .
$$

The time-reversible "friction coefficient" $\zeta$ which follows from Hamilton's Principle is equal to the rate at which heat is extracted to satisfy the constraint, divided by twice the kinetic energy $K$ of the constrained degrees of freedom:

$$
\zeta_{\text {HAMILTON }}=-\dot{Q} / 2 K \text {. }
$$

Thus $\zeta$ corresponds to the rate at which entropy is absorbed by a reservoir modeled by the constraint. It was only recently discovered that this correspondence follows directly from Hamilton's Principle. Exactly the same equation of motion (1) follows also from the simple expedient of velocity rescaling or from the more-elegant, but equivalent, application of Gauss' Principle of Least Constraint to the problem of thermostatting mechanical motions [6, 7],

An alternative route to incorporating temperature into mechanics was discovered by Nose [8], He too found exactly the same equation of motion as follows from Hamilton's Principle, but with a somewhat different reversible friction coefficient:

$$
\zeta_{\text {NOSÉ-HOOVER }}=\int(2 \Delta K / k T) / \tau^{2} d t .
$$

Here $k T$ is twice the equilibrium equipartition kinetic energy of a thermostatted degree of freedom and $\Delta \mathrm{K}$ is the instantaneous deviation of the total kinetic energy from the average value, $\mathrm{x}$ is an arbitrary relaxation time, which in practice is best chosen such that the timescale of the energy fluctuations corresponds to the time between collisions for a typical thermostatted particle. In the equilibrium case $\zeta_{\mathrm{N}-\mathrm{H}}$ has a Gaussian distribution, increasing the number of phase-space coordinates by one. The additional variable is the 
Nosé-Hoover friction coefficient $\zeta_{\mathrm{N}-\mathrm{H}}$. Nosé chose $\zeta_{\mathrm{N}-\mathrm{H}}$ so as to reproduce Gibbs' equilibrium canonical distribution for the phase-space variables $\{q, p\}$. I have championed the particular version of his mechanics, "Nose-Hoover mechanics", which avoids "timescaling" of the constrained motions.

The half-width of the probability distribution for the friction coefficient $\zeta$ is proportional to $1 / \tau$. In the limit that $\tau$ approaches zero, Nose's mechanics reproduces the same constrained dynamics which follows from Hamilton's Principle of Least Action. There is so far no derivation of Nose-Hoover friction based on a variational principle.

These thermostatting forces, with either Hamiltonian or Nose-Hoover friction coefficients, have been employed in a variety of simulations, both at, and away from, equilibrium. They made it possible to use temperature as an independent variable in computer simulations of small and large systems. Generalizations have also been used to control the internal energy as well as nonequilibrium fluxes. It should be emphasized that the time-reversibility of mechanics is retained under the influence of thermostatting. Both formulations just mentioned are fully time-reversible, with the friction coefficient changing sign in the reversed motion.

\section{LYAPUNOV INSTABILITY IN MANY-BODY SYSTEMS $[6,7,9]$}

The exponential increase of small perturbations $\{\delta q, \delta p\}$ in the initial conditions,

$$
\delta(t) / \delta(0) \approx \exp \left(\lambda_{1} t\right)
$$

is a common feature of solutions of the many-body problem. This increase, which leads formally to divergence at long times, is quantified, and generalized, through the Lyapunov exponents, both the "local" instantaneous exponents $\{\lambda(t)\}$ and the "global" timeaveraged exponents $\{\langle\lambda(t)\rangle\} \equiv\{\lambda\}$, which describe the comoving and corotaing deformations of a phase-space hypersphere governed by deterministic equations of motion. Both of the thermal generalizations of mechanics outlined in Section 2 provide the same link between the Lyapunov spectrum, the friction coefficient, or coefficients, and the resulting heat transferred by the reversible friction forces:

$$
\sum \lambda(t)=-\sum \zeta=\sum(\dot{Q} / k T)
$$

Here the complete Lyapunov spectrum is summed up, over all degrees of freedom, including the additional degree of freedom $\zeta_{\mathrm{N}-\mathrm{H}}$ in the Nosé-Hoover case. The friction coefficient and heat-transfer sums include only those degrees of freedom affected by the reversible friction forces, together with the temperatures of the reservoirs to which they are coupled. In the usual case the time-rate-of-change of $Q$ is negative, corresponding to heat flow from the system to the surroundings:

$$
\sum \lambda(t)=-\sum \zeta=\sum(\dot{Q} / k T)<0 .
$$

The key step in establishing this result is the phase-space flow equation, the analog of Liouville's Theorem:

$$
d \ln f / d t=-\sum \zeta=-d \ln \otimes / d t
$$

a local identity which follows directly from the thermostatted equations of motion. 


\section{CLAUSIUS' INEQUALITY AND LYAPUNOV INSTABILITY}

This relationship between the Lyapunov exponents and the heat transfer shows that no process which absorbs an infinite amount of heat can take place in a bounded region of phase space. Likewise, any process which gives off an infinite amount of heat must occupy a vanishingly small region of phase space. Evidently any cyclic process which absorbs or generates heat must, when repeated, lead either to unbounded or to vanishing phase-space hypervolume.

Thus the requirement that averages over the phase-space distribution be bounded excludes cyclic processes which absorb heat, in accord with Clausius' inequality, and allows only those processes which correspond to a vanishing region of the equilibrium phase space. Extensive numerical work has established that the allowed phase space regions are multifractal strange attractors, with an information dimension strictly less than that of the equilibrium phase-space distribution, corresponding to local Lyapunov spectra $\{\lambda(t)\}$ whose time-averaged global sums are strictly negative:

$$
\sum \lambda<0 \text {. }
$$

Thus the ideal-gas definition of temperature, coupled with thermostats based on Hamilton's principle or Gibbs' distribution, makes possible the analysis of processes involving heat transfer, and an explanation of thermodynamic irreversibility in terms of the dynamic Lyapunov instability of the microscopic motion equations. This point of view provides no support for the notion of a nonequilibrium entropy and suggests instead that entropy is a useful concept only at equilibrium. The loss of the entropy concept away from equilibrium is not a serious one, as Onsager's symmetry results can be equally well obtained from Green and Kubo's nonentropic approach. The multifractal nature of the nonequilibrium phase-space distributions which result from the use of thermostats give Gibbs' entropies of $-\infty$, corresponding to the extremely low probability of randomly encountering a typical nonequilibrium state. Such typical nonequilibrium states have a past history which is not at all typical of equilibrium systems.

This thermomechanical explanation of irreversible behavior is more general than Boltzmann's, for it is not restricted to gases, and it is also more general than Green and Kubo's, because it is not restricted to linear deviations from equilibrium. It shows that the dynamical instability associated with the many-body problem requires Clausius' inequality. Otherwise, thermal instability would cause thermomechanical phase-space averages to diverge.

\section{CONTINUUM MECHANICS SIMULATIONS USING SMOOTH PARTICLES}

A new approach to computational continuum mechanics was originated by Lucy and Monaghan in 1977 [3], Their idea uses "smooth particles" to describe the time development of the continuum field variables. This new version of continuum mechanics associates individual velocities and energies with each particle but calculates the field variables at any point in space, $r$, by superposing the contributions of all particles within range of the smoothing function $w(r)$

$$
\langle v\rangle_{r}=\sum m w_{r i} v_{i} / \rho_{i} ;\langle e\rangle_{r}=\sum m w_{r i} e_{i} / \rho_{i} ; \rho_{i}=\sum m w_{i j} .
$$


The smoothing function $w$ is generally chosen to have continuous first and second derivatives and a finite range, over which its integral is unity. The equation of motion associated with smooth particle applied mechanics includes the stress tensors and densities of all pairs of particles close enough to interact:

$$
m \ddot{r}_{i}=\sum m^{2}\left[\left(\sigma / \rho^{2}\right)_{i}+\left(\sigma / \rho^{2}\right)_{j}\right] \cdot \nabla_{i} w_{i j} .
$$

The stress tensors and densities at particles $\mathrm{i}$ and $\mathrm{j}$ are to be calculated by summing up the contributions from all nearby particles, just as was done in the example calculation of the velocity $\langle v\rangle$ above.

The special case appropriate to a two-dimensional ideal-gas isentrope,

$$
\sigma \propto \rho^{2}
$$

reduces exactly to Newton's equations of motion with the smooth particle weight function playing the role of a pair potential. This similarity suggests that the evolution of a continuum shares the Lyapunov instability that characterizes atomistic flows. It suggests also that efforts to describe the interaction of eddies in turbulent flow are equivalent to the description of the coupling of velocity fluctuations in a correspondingly driven atomistic flow. Ongoing work, carried out with Oyeon Kum, Harald Posch, and Carol Hoover, should shed light on this second link between microscopic mechanics and macroscopic nonequilibrium processes.

\section{Acknowledgment}

I am grateful to the Universities of Berlin, California, and Vienna for the opportunity to carry out this work, to Krzysztof Wojciechowski, at the Institute for Molecular Physics in Poznan, and to Harald Posch, in the Institute for Experimental Physics at the University of Vienna, for further support and stimulation. The ideas underlying this work have additionally been discussed, over a period of years, in many stimulating conversations with Brad Holian (Los Alamos), Carol Hoover (Livermore), and Oyeon Kum (Korean Defense Agency). Work carried out at the University of California was supported by Department of Energy Contract W-7405-Eng-48 between the University of California and the Lawrence Livermore National Laboratory.

\section{References}

[1] W. Muschik, Am. J. Phys. 58, 241 (1990).

[2] P. Van and W. Muschik, Phys. Rev. E 52, 3584 (1995).

[3] J. J. Monaghan, Ann. Rev. Astron. and Astrophys. 30, 542 (1992).

[4] O. Kum, H. A. Posch, and W. G. Hoover, Phys. Rev. E 52, 1711, 4899 (1995).

[5] W. G. Hoover, Phys. Letts. A 204, 133 (1995).

[6] W. G. Hoover, Computational Statistical Mechanics (Elsevier, Amsterdam, 1991.

[7] D. J. Evans and G. P. Morriss, Statistical Mechanics of Nonequilibrium Liquids (Academic, New York, 1990). See also the reviews of Hoover and Evans in the Proceedings of StatPhys 18, published as volume 194 of Physica A (1993).

[8] S.Nosé, J. Chem. Phys. 81,511 (1984).

[9] W. G. Hoover and H. A. Posch, Phys. Rev. E 49, 1913 (1994). 